\title{
DIMENSIONAMENTO DA CÂMARA DE SECAGEM DE SPRAY DRYER PARA PRODUÇÃO DE FARINHA DE BANANA VERDE
}

\author{
R. K. OI ${ }^{1}$, E. B. TAMBOURGI ${ }^{2}$ e D.de MORAES $\mathrm{Jr}^{3}$ \\ ${ }^{1}$ Universidade Católica de Santos \\ ${ }^{2}$ Universidade Estadual de Campinas \\ ${ }^{3}$ Universidade Santa Cecília \\ e-mail para contato: prof_oi@ymail.com
}

\begin{abstract}
RESUMO - Os consumidores estão buscando, cada vez mais, alimentos que além de cumprir as funções nutricionais básicas também proporcionam efeitos benéficos à saúde.A farinha de banana verde surge com uma opção para ser utilizada em substituição à farinha de trigo na elaboração de massas ou como espessante alimentar, pois assume o sabor e o aroma dos alimentos elaborados a partir dela. Na banana verde está presente o amido resistente, que apresenta diversos efeitos benéficos sobre alguns males como câncer coloretal, diarréia, índice glicêmico, dislipidemias, doenças cardiovasculares e doença celíaca. O spray dryer é o equipamento mais versátil para a secagem de gêneros alimentícios, permitindo obter produtos na forma de pó. Este trabalho apresenta um estudo para o dimensionamento da câmara de secagem de um spray dryerpiloto para a produção de $25 \mathrm{~kg} / \mathrm{h}$ de farinha de banana verde através dos dados obtidos em um spray dryer experimental, em que foram utilizadas as variáveis: rotação do atomizador; temperatura da alimentação e a vazão da alimentação. Estabeleceu-se como resposta a massa do produto seco. O dimensionamento da câmara de secagem do spray dryer piloto foi desenvolvido a partir do ensaio em que se obteve a maior quantidade de massa.
\end{abstract}

\section{INTRODUÇÃO}

A necessidade de melhoria da saúde leva os consumidores a buscar alimentos específicos ou componentes alimentares fisiologicamente ativos, também denominados alimentos funcionais. Nos últimos anos surgiram diversas opções para essa categoria de alimentos, dentre essas a biomassa de banana verde.

A banana verdecontém alto teor de amido, cerca de $20 \%$ e, desse total, dependendo da espécie, até $84 \%$ pode se encontrar na forma de amido resistente (FREITAS e TAVARES, 2005). O amido resistente é uma forma de amido resultante da degradação do amido não absorvido pelo intestino delgado e apresenta características semelhantes das fibras insolúveis, ajudando a regular o trabalho intestinal (SALGADO et al, 2005). 
Muitos trabalhos foram publicados sobre as propriedades da banana verde, que abordaram os efeitos benéficos sobre males como câncer coloretal, diarréia, índice glicêmico, resposta insulínica, dislipidemias, doenças cardiovasculares e doença celíaca (ZANDONADI, 2009; TOPPING, FUKUSHIMA e BIRD, 2003; FREITAS e TAVARES, 2005).

Essa biomassa é obtida pela cocção e trituração da banana verde, ficando esse produto na forma pastosa, ausente de sabor e inodora. A biomassa de banana verde pode ser utilizada como substituta dos espessantes tradicionais como trigo, soja, fécula de mandioca e amido de milho, melhorando o valor nutricional e assumindo o sabor e o aroma dos alimentos preparados com essa substância. Outra utilização é na preparação de massas alimentícias.

A biomassa de banana verde ainda é produzida artesanalmente ou semi-industrialmente, em baixa escala de produção e obtida sob a forma pastosa, e mesmo que o processamento seja asséptico, persiste a preocupação quanto à questão microbiológica e de perecibilidade do produto. Uma alternativa é a secagem dessa biomassa e sua obtenção em forma de pó.

A secagem é a transferência de um líquido que se encontra em um sólido molhado para uma fase gasosa não saturada (FOUSTet al., 1982).Na operação de secagem são empregados secadores industriais conforme as características da substância a ser seca e a qualidade do produto final. Os secadores são desenvolvidos a partir de algumas condições de secagem como estática, dinâmica, fluidizada e diluída (PERRY e GREEN, 1997).

A secagem na condição diluída ocorre através da atomização, que é o processo de divisão do líquido em milhões de micro gotas formando um spray, sendo que $1 \mathrm{~m}^{3}$ de líquido forma cerca de 2 x $10^{12}$ gotas com diâmetro aproximado de $100 \mu \mathrm{m}$ (MASTERS, 1985).

O secador spray dryer funciona pelo principio da secagem diluída, aceitando fluidos bombeáveis na entrada da alimentação até o final do processo e produz partículas secas, sem a necessidade de se empregar elevadas temperaturas, que podem degradar os nutrientes do produto a ser seco (MASTERS, 1985). Esse tipo de secador é amplamente utilizado na secagem industrial de diversos produtos, incluindo os alimentícios. As propriedades físicoquímicas dos produtos obtidos secos em spray dryersão preservadas (FOUSTet al., 1982).

A secagem da biomassa de banana verde em spray dryer permite a remoção da água e a granulação do material, facilitando o transporte e aumentando a vida de prateleira do produto. Oiet al. (2013), Oiet al. (2012a, 2012b), Oiet al. (2010) e Oiet al. (2009) lograram êxito na secagem de biomassa de banana verde em spray dryer.

O presente trabalho teve por objetivo dimensionar a câmara de secagem de um spray dryerpiloto para a produção de $25 \mathrm{~kg} / \mathrm{h}$ de farinha de banana verde, a partir dos dados obtidos de ensaios realizados em um spray dryer experimental, sendo utilizada as variáveis rotação do atomizador, vazão da alimentação e temperatura da alimentação.

A determinação do diâmetro da câmara de secagem é o item mais relevante para o projeto de um secador spray dryer, pois a partir dele define-se a especificação das demais partes, sendo que grande parte dessas podem ser encontradas comercialmente nos catálogos de fabricantes especializados, justificando-se o desenvolvimento deste estudo. 


\section{MATERIAIS E MÉTODOS}

Os ensaios para o estudo da secagem da pasta de banana verde foram realizados em um spray dryer não comercial, que está representado pela Figura 1. O equipamento é constituído de uma câmara de secagem com volume de $0,2 \mathrm{~m}^{3}$, sendo o diâmetro de $0,63 \mathrm{~m}$ e a altura de $0,91 \mathrm{~m}$, fabricada em aço carbono e revestida internamente com resina polimérica (epóxi). Esse equipamento experimental foi construído especialmente para o desenvolvimento dos ensaios com a pasta de banana verde.

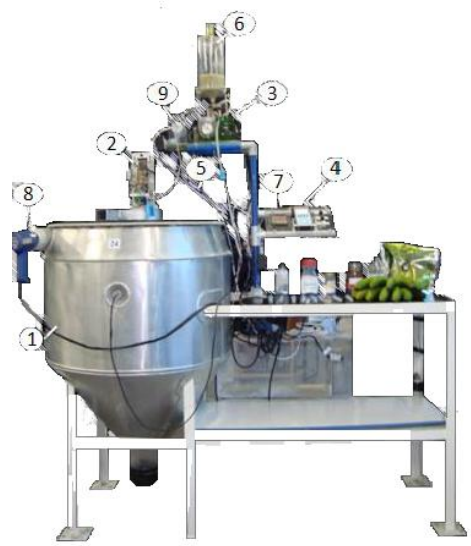

1) Câmara de secagem; 2) Motor elétrico; 3) Bomba peristáltica; 4) Indicador de temperatura; 5) Mangueiras de silicone para o transporte da suspensão; 6) Tanque de alimentação; 7) Controlador de vazão volumétrica; 8) Aquecedor de ar; 9) Trocador de calor.

Figura 1 - Unidade experimental de spray dryer

$\mathrm{O}$ atomizador rotativo foi movimentado por um motor elétrico retirado de uma retífica da marca LEE de potência $127 \mathrm{~W}$, com controle de rotação e arrefecido por um cooler.A suspensão foi injetada no atomizador por uma bomba peristáltica constituída de um rotor de alumínio com quatro roletes, e acionado pelo motor elétrico. A suspensão foi armazenada em um tanque de formato cilíndrico fabricado em acrílico. Na tampa superior foi fixado um agitador movimentado por um motor elétrico para homogeneização da alimentação.

A suspensão foi aquecida, antes de ser atomizada, em um trocador de calor com resistência elétrica, que aquece a parede externa do tubo da alimentação, revestida com isolante térmico e protegida por uma carcaça de alumínio. $\mathrm{O}$ ar foi aquecido por dois aquecedores de resistência elétrica, instalados na câmara em lados opostos, da marca Steinel, modelo HL1800E, com potência de $2.000 \mathrm{~W}$ cada. O painel de controle utilizado foi da marca Salviterm, modelo 704 com quatro canais.

Na pasta de banana verde, foi adicionada água para evitar a obstrução no atomizador e nos dutos do equipamento. Assim, nos ensaios foi utilizada uma suspensão de pasta de banana e água na concentração de $50 \%$ de sólidos, estabelecida após ensaios preliminares.

No experimento foram utilizadas como variáveis a rotação do atomizador, a vazão da alimentação e a temperatura da alimentação, em dois níveis de variação, o que correspondeu à realização de oito ensaios $\left(2^{3}\right)$, seguindo um planejamento experimental. A Tabela 1 apresenta as variáveis e seus níveis de variação. 
Tabela 1 - Valores das variáveis utilizadas nos ensaios.

\begin{tabular}{l|l}
\hline Variáveis & Valores \\
\hline \multirow{2}{*}{ Rotação do eixo do motor } & $23.000 \mathrm{rpm}$ \\
\cline { 2 - 2 } $\begin{array}{l}\text { Temperatura de } \\
\text { pré-aquecimento }\end{array}$ & $30^{\circ} \mathrm{C}$ \\
\cline { 2 - 2 } & $40^{\circ} \mathrm{C}$ \\
\hline \multirow{2}{*}{ Vazão de alimentação } & $40 \mathrm{~mL} / \mathrm{min}$ \\
\cline { 2 - 2 } & $60 \mathrm{~mL} / \mathrm{min}$ \\
\hline
\end{tabular}

As temperaturas do ar na entrada e na saída foram fixadas em todos os ensaios, sendo os valores, respectivamente, $22^{\circ} \mathrm{C}$ e $160^{\circ} \mathrm{C}$.A partir da regulagem do spray dryer nas condições de cada ensaio, coletou-se o produto durante 15 minutos. Em seguida as amostras foram pesadas em uma balança de resolução $10^{-3} \mathrm{~g}$ da marca Marte, modelo AL 500, registrando-se a massa das amostras.

No dimensionamento da câmara foi utilizado o maior valor de massa dentre as oito amostras obtidas no experimento. A seguir são apresentadas as equações utilizadas para o dimensionamento da câmara de secagem do spray dryer, conforme Oiet al (2009).

O cálculo do volume da câmara de secagem é dado pela equação 1:

$$
V=\tau \cdot Q_{c}
$$

Sendo: $\tau$ o tempo espacial e $Q_{c}$ a vazão volumétrica na câmara, definida pela equação 2:

$$
Q_{c}=\frac{\omega_{\text {arnatemp de saida }}}{\rho_{\text {arnatemp de saida }}}
$$

Em que $\rho$ é a massa específica do ar na temperatura de saída e $\omega$ a vazão mássica do ar na temperatura de saída, que pode ser determinada pela equação 3:

$$
\omega_{\text {ar natemp de saida }}=\omega_{\text {produto }} \cdot x
$$

A variável $\omega_{\text {produto }}$ representa a vazão mássica do produto seco (pó da pasta de banana verde) e x (equação 4) a relação entre as vazões mássicas de ar na temperatura de entrada e do produto, obtida na unidade experimental com as variáveis otimizadas.

$$
x=\frac{\omega_{\text {ar na temp de entrada }}}{\omega_{\text {produto }}}
$$

A vazão mássica do ar na temperatura de entrada pode ser determinada pela equação 5, em que v é a velocidade medida através de um anemômetro e A a área da seção transversal na entrada do secador.

$$
\omega_{\text {ar na temp de entrada }}=\rho_{\text {ar na temp de entrada }} \cdot v \cdot A
$$


A massa específica do ar $\rho$ pode ser determinada através da equação 6 , sendo $p$ a pressão absoluta, $\mathrm{M}$ a massa molar do ar, $\mathrm{R}$ a constante dos gases e $\mathrm{T}$ a temperatura do ar.

$$
\rho=\frac{p \cdot M}{R \cdot T}
$$

Segundo Oiet al (2009), a partir do cálculo do volume da câmara pela equação 1, determina-se o diâmetro interno por meio da equação 7:

$$
V=\frac{\pi}{4} \cdot D^{2} \cdot\left(H+\frac{\sqrt{3}}{2} \cdot D\right) \Rightarrow D=\sqrt[3]{\frac{V}{1,47}}
$$

\section{3 - RESULTADOS E DISCUSSÃO}

A Tabela 2 apresenta os resultados dos oito ensaios realizados usando o spray dryer experimental com atomizador rotativo, em que: $\mathrm{n}$ é a rotação do atomizador; $\mathrm{T}$ é a temperatura de alimentação; Q é a vazão de alimentação e m é a massa do pó da banana verde, coletada durante os 15 minutos de cada ensaio.

Tabela 2 - Ensaios realizados no spray dryer

\begin{tabular}{l|crrc}
\hline Ensaio & $\begin{array}{c}\boldsymbol{n} \\
(\boldsymbol{r p m})\end{array}$ & $\begin{array}{c}\boldsymbol{T} \\
\left({ }^{\boldsymbol{C}} \boldsymbol{C}\right)\end{array}$ & $\begin{array}{c}\boldsymbol{Q}(\boldsymbol{m} \boldsymbol{L} / \\
\boldsymbol{m i n})\end{array}$ & $\begin{array}{c}\boldsymbol{m} \\
(\boldsymbol{g})\end{array}$ \\
\hline 1 & 23000 & 30 & 40 & 11,452 \\
2 & 23000 & 30 & 60 & 11,507 \\
3 & 23000 & 40 & 40 & 12,016 \\
4 & 23000 & 40 & 60 & 12,739 \\
5 & 27000 & 30 & 40 & 12,656 \\
6 & 27000 & 30 & 60 & 18,295 \\
7 & 27000 & 40 & 40 & 37,400 \\
8 & 27000 & 40 & 60 & 37,560 \\
\hline
\end{tabular}

Dentre as oito amostras, aquela produzida pelo ensaio $\mathrm{n}^{\circ} 8$ apresentou a melhor quantidade de massa, $37,560 \mathrm{~g}$, sendo obtida com as seguintes variáveis: rotação de 27.000 $\mathrm{rpm}$; temperatura de alimentação de $40^{\circ} \mathrm{C}$ e vazão de alimentação de $60 \mathrm{~mL} / \mathrm{min}$.

A partir do valor da massa dessa amostra foi dimensionada a câmara de secagem do spray dryer para a produção de $25 \mathrm{~kg} / \mathrm{h}$ de farinha de banana verde. No ensaio $\mathrm{n}^{\mathrm{o}} 8$ o teor de água da amostra foi de $6 \%$. O teor de água na pasta de banana verde era de $70 \%$.

A massa específica do ar na temperatura de entrada $\left(22^{\circ} \mathrm{C}, 295,15 \mathrm{~K}\right)$ foi calculada através da equação 6 e considerando os seguintes valores:

$$
\begin{aligned}
& \mathrm{M}_{\mathrm{N} 2}=28 \mathrm{~g} / \mathrm{mol} \mathrm{M}_{\mathrm{O} 2}=32 \mathrm{~g} / \mathrm{mol} \\
& \bar{M}=0,79 \cdot 28+0,21 \cdot 32 \rightarrow \bar{M}=28,84 \mathrm{~g} / \mathrm{mol}
\end{aligned}
$$




$$
\rho_{\text {ar natemp de entrada }}=\frac{1 \mathrm{~atm} \cdot 28,84 \mathrm{~g} / \mathrm{mol}}{0,082 \frac{\mathrm{atm} \cdot \mathrm{l}}{\mathrm{mol} \cdot \mathrm{K}} \cdot 295,15 \mathrm{~K}} \rightarrow \rho_{\text {arnatemp de entrada }}=1,19 \frac{\mathrm{g}}{\mathrm{l}}=1,19 \frac{\mathrm{kg}}{\mathrm{m}^{3}}
$$

A massa específica do ar na temperatura de saída $\left(160^{\circ} \mathrm{C}, 433,15 \mathrm{~K}\right)$ foi calculada através da equação 6 .

$$
\rho_{\text {ar na temp de saida }}=\frac{1 \mathrm{~atm} \cdot 28,84 \mathrm{~g} / \mathrm{mol}}{0,082 \frac{\mathrm{atm} \cdot \mathrm{l}}{\mathrm{mol} \cdot \mathrm{K}} \cdot 433,15 \mathrm{~K}} \rightarrow \rho_{\text {ar natemp de saida }}=0,81 \frac{\mathrm{g}}{\mathrm{l}}=0,81 \frac{\mathrm{kg}}{\mathrm{m}^{3}}
$$

Da equação 5 e considerando a área da seção transversal $\mathrm{A}=5,28.10^{-3} \mathrm{~m}^{2}$ (dois aquecedores) e $\mathrm{v}=2,2 \mathrm{~m} / \mathrm{s}$ (medido em anemômetro digital) tem-se, para o secador:

$$
\omega_{\text {ar na temp de entrada }}=1,19 \frac{\mathrm{kg}}{\mathrm{m}^{3}} \cdot 2,2 \frac{\mathrm{m}}{\mathrm{s}} \cdot 5,28 \cdot 10^{-3} \mathrm{~m}^{2} \rightarrow \omega_{\text {ar natemp de entrada }}=1,384 \cdot 10^{-2} \frac{\mathrm{kg}}{\mathrm{s}}
$$

O cálculo da relação entre as vazões mássicas de ar na temperatura de entrada e do produto ensaiado $(37,560 \mathrm{~g})$ é obtido através da equação 4:

$$
x=\frac{1,384 \cdot 10^{-2} \mathrm{~kg}_{\text {ar }} / \mathrm{s}}{\frac{37,560 \mathrm{~g}_{\text {produto }} \cdot 1 \mathrm{~kg} / 1000 \mathrm{~g}}{15 \mathrm{~min} \cdot 60 \mathrm{~s} / \mathrm{min}}}=331,674 \frac{\mathrm{kg}_{\text {ar }}}{\mathrm{kg}_{\text {produto }}}
$$

Para a produção desejada de $25 \mathrm{~kg} / \mathrm{h}$ do produto seco, da equação 3 determina-se vazão mássica do ar na temperatura de saída.

$$
\omega_{\text {ar na temp de saida }}=25 \frac{\mathrm{kg}_{\text {produto }}}{\mathrm{h}} \cdot \frac{1 \mathrm{~h}}{3600 \mathrm{~s}} \cdot 701,45 \frac{\mathrm{kg}_{\text {ar }}}{\mathrm{kg}_{\text {produto }}}=2,303 \frac{\mathrm{kg} \text { ar }}{\mathrm{s}}
$$

Por meio da equação 2 calcula-se a vazão volumétrica:

$$
Q_{c}=\frac{2,303 \mathrm{~kg}_{a r} / \mathrm{s}}{0,81 \mathrm{~kg}_{a r} / \mathrm{m}^{3}}=2,837 \frac{\mathrm{m}^{3}}{\mathrm{~s}}
$$

Com a equação 1 determina-se o volume da câmara. Foi considerado o tempo espacial de $25 \mathrm{~s}$, valor estimado através de experiência em projetos (PERRY e GREEN, 1997).

$$
V=25 s \cdot 2,837 m^{3} / s=70,916 m^{3}
$$

A partir da equação 7 obtém-se o diâmetro da câmara de secagem (OI et al., 2009).

$$
D=\sqrt[3]{\frac{70,916}{1,47}}=3,640 m
$$


A dimensão determinada $(\mathrm{D}=3,640 \mathrm{~m})$ corresponde ao diâmetro interno da câmara de secagem. O material da parede interna deve ser de aço inoxidável usado no processamento de alimentos. Já a parede externa pode ser feita com liga de alumínio, sendo que o espaço entre as paredes deve ser preenchido com material isolante, tendo em vista reduzir a perda de calor pela parede do secador, aumentando-se a eficiência do equipamento. A altura da câmara é determinada através do seguinte cálculo:

$$
H=3,640+\tan 60^{\circ} \times \frac{3,640}{2} \rightarrow H=6,793 m
$$

A Figura 2 ilustra a câmara de secagem do spray dryer, sendo altura do cilindro igual ao diâmetro e o ângulo do cone reto de 60 (PERRY e GREEN, 1997).

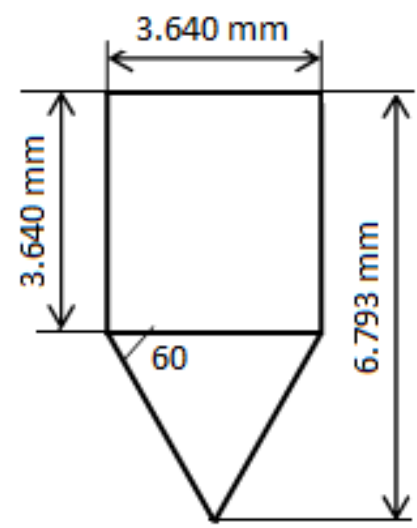

Figura 2 - Câmara de secagem do spray dryer

\section{CONCLUSÃO}

O diâmetro da câmara de secagem do spray dryer para produzir $25 \mathrm{~kg} / \mathrm{h}$ de farinha de banana verde deve ser de 3,640 m e a altura de 6,793 $\mathrm{m}$. A altura total do equipamento deve considerar o coletor do produto e a estrutura de sustentação na parte inferior, o que corresponderá à dimensão aproximada de $8 \mathrm{~m}$.

Dessa forma, o pé direito mínimo da planta industrial deve ser de $10 \mathrm{~m}$, tendo em vista o espaço para a manutenção do equipamento. Para maiores volumes de produção, o método para o dimensionamento da câmara seria mantido, inclusive a forma de injeção da alimentação, pela ampliação de escala.

Para a produção de $25 \mathrm{~kg} / \mathrm{h}$ de farinha de banana verde, considerando dois turnos de trabalho por dia, com seis horas úteis de trabalho cada (o tempo restante é destinado ao set-up do equipamento e outros tempos ociosos), a produção diária é de $150 \mathrm{~kg}$. Num mês com 22 dias úteis representa uma produção mensal de 6.600 kgpor equipamento.

Deve-se considerar uma escala de produção de farinha de banana verde que compense o elevado investimento para a construção de um equipamento de spray dryer e seus custos operacionais,portanto, recomenda-se desenvolver um estudo de viabilidade econômica. 


\section{REFERÊNCIAS}

FOUST, A. S.; WENZEL, L. A.; CLUMP, C. W.; MAUS, L.; ANDERSEN, L. B. Princípios das Operações Unitárias. 2ª ed. Rio de Janeiro: Ed. Guanabara Dois, 1982.

FREITAS, M. C. J.; TAVARES, D. Q. Caracterização do grânulo de amido de banana (Musa AAA-Nanicão e Musa AAB Terra). Revista Ciência e Tecnologia de Alimentos, Campinas, v. 25, n. 2, p. 217-222, 2005.

MASTERS, K. Spray Drying Handbook. 4th. ed. London: George Godwin, 1985.

OI, Ricardo K.; SANTANA, J. C. C.; TAMBOURGI, E. B.; MORAES JR, D. Feasibility Study for Production of Green Banana Flour in a Spray Dryer. Chemical Engineering Transactions, v. 32, p. 1825-1830, 2013.

OI, Ricardo K.; TAMBOURGI, E. B. ; MORAES JR, D. Estudo da Secagem da Biomassa de Banana Verde em Spray Dryer. Engevista (UFF), v. 14, p. 165-171, 2012.

OI, Ricardo K.; MORAES JR, D.; TAMBOURGI, E. B. Estudo de viabilidade para produção da farinha de banana verde em spray dryer. Revista Brasileira de Produtos Agroindustriais, v. 14, p. 317-322, 2012.

OI, Ricardo K.; TAMBOURGI, E. B.; MORAES JR, D. Estudo de viabilidade da secagem da biomassa da banana verde em spray dryer rotativo. Exacta (São Paulo. Impresso), v. 8, p. online, 2010.

OI, R. K.; MORAES, M. S.; FERNANDES, F. M.; MOINO, C. A. A.; TAMBOURGI, E. B.; MORAES JR, D. Projeto de secador do tipo spray dryer para secagem de biomassa de banana verde. In: IX Congreso Iberoamericano de Ingeniería Mecánica, Las Palmas de Gran Canária: Airexpress, 2009.

PERRY, R. H.; GREEN, D. W. Perry's Chemical Engineers' Handbook. 7th. ed. New York: McGraw-Hill, 1997.

SALGADO, S. M.; DE FARO, Z. P.; GUERRA, N. B.; LIVERA, A. V. S. Aspectos físicoquímicos e fisiológicos do amido resistente. Boletim do CEPPA, v. 23, n. 1, p. 109-122, 2005.

TOPPING, D. L.; FUKUSHIMA, M.; BIRD, A. R. Resistant starch as a prebiotic and synbiotic. Proceedings of the Nutrition Society, v. 62, p. 171-176, 2003.

ZANDONADI, R. P. Massa de banana verde: uma alternativa para exclusão do glúten. 2009. 74f. Tese (Doutorado em Ciências da Saúde), Faculdade de Ciências da Saúde, Universidade de Brasília - UnB, Brasília. 